\title{
Evaluación de la ceniza agroindustrial de palma aceitera en suelos ácidos del Pacífico Sur, Costa Rica
}

\author{
Ángel Madrigal Valverde ${ }^{1 *}$ Alexander Palacios Cascante ${ }^{2}$ Gabriel Garbanzo León ${ }^{3}$.
}

${ }^{1}$ Unidad Operaciones Regionales Brunca, Servicio Fitosanitario del Estado, Ministerio de Agricultura y Ganadería. Golfito, Puntarenas, Costa Rica.*Autor para correspondencia: amadrigal@sfe.go.cr

2 Departamento de Investigación y Desarrollo Palma Tica S. A, Corredores, Puntarenas, Costa Rica. apalacios@palmatica.com

3 Centro de Investigaciones Agronómicas, Escuela de Agronomía, Universidad de Costa Rica. juan.garbanzo@ucr.ac.cr.

Recibido: 12 de agosto de 2020 Aceptado: 22 de octubre de 2020

RESUMEN: Se evaluó el efecto del residuo industrial en la variedad Deli x Ghana en una plantación comercial. Para esto se aplicó distintas dosis de este material (5000 kg ha-1, $10000 \mathrm{~kg} \mathrm{ha}^{-1}$ y $15000 \mathrm{~kg} \mathrm{ha}{ }^{-1}$ ).Las aplicaciones se realizaron en las rodajas de las palmas, siguiendo un diseño experimental de bloques completos al azar. Se midió la concentración de nutrimentos en el suelo, densidad de raíz y la conductancia estomática en hojas 9 y 17, a los 90 y 180 días después de la aplicación de los tratamientos (dda). Las mediciones a nivel del suelo, mostraron una tendencia en el aumento del $\mathrm{pH}$ y la disminución de la acidez intercambiable, a los 180 dda. También se presentó un aumento en la densidad de raíz a los 90 dda en las palmas del tratamiento en el que se adicionó $10000 \mathrm{~kg} \mathrm{ha}^{-1}$ (C10) y a los $180 \mathrm{dda}$, en respuesta a la aplicación de $5000 \mathrm{~kg} \mathrm{ha}^{-1}$ y 15000 $\mathrm{kg} \mathrm{ha}^{-1}$ (C5 y C15). La conductancia estomática, medida en la hoja $\mathrm{N}^{\circ} 9$ antes del mediodía, mostró diferencia estadística significativa en los tratamientos C5, C10 y C15. Se concluye que la adición de ceniza industrial de palma aceitera tiene un efecto positivo en la variación de la conductancia estomática y la densidad de raíces en palma aceitera.

Palabras claves: Cenizas industrial, Deli x Ghana, Densidad de raíz, conductancia estomática.

\begin{abstract}
Evaluation of ash residue from the oil extracting plant in oil palm (Elaeis guineensis) in acid soils of south pacific, Costa Rica. It was evaluated the effect of industrial residue on the Deli $x$ Ghana variety in a commercial plantation. Different increasing doses $\left(5000 \mathrm{~kg} \mathrm{ha}^{-1}, 10000 \mathrm{~kg} \mathrm{ha}^{-1}\right.$ and $15000 \mathrm{~kg} \mathrm{ha}$ $\left.{ }^{1}\right)$ was applied. This was located at the palm slices in a randomized complete block design. Nutrient soil concentration, root density and stomatic conductance were evaluated at 90 and 180 days after their application (dda). It was found that after 180 dda that $\mathrm{pH}$ increased and decreasing soil acidity. Root density also increased at $90 \mathrm{dda}$ with the addition of $10000 \mathrm{~kg} \mathrm{ha}^{-1}$ (C10) and at $180 \mathrm{dda}$ with the application of $5000 \mathrm{~kg} \mathrm{ha}^{-1}$ and 15 $000 \mathrm{~kg} \mathrm{ha}^{-1}$ (C5 and C15). Stomatic conductance showed significant statistical difference in treatments C5, C10 and $\mathrm{C} 15$ on sheet $\mathrm{N}^{\circ} 9$ at noon. It was concluded addition of industrial oil palm ash had a positive effect on the variation of stomatic conductance in oil palm and root density.
\end{abstract}

Keywords: Industrial ash, Deli x Ghana, root density, stomatal conductance. 


\section{Introducción}

La palma aceitera (Elaeis guineenis Jacq) es una oleaginosa que se ha expandido considerablemente alrededor del mundo (Escobar 2012 y Trucchi 2015). La principal razón de este crecimiento obedece a que E. guineensis presenta un mayor rendimiento en la producción de fruta fresca por hectárea, siendo un cultivo muy llamativo para los agricultores por su alta producción de aceite y bajos insumos agrícolas (Sánchez 2003 y Madrigal et al. 2018). A nivel mundial cerca de 22506550 ha se habían establecido en el 2014, principalmente en países como: Malasia, Indonesia, Tailandia y Nigeria. Estos países representan el $81,7 \%$ de la producción mundial (Sierra et al. 2017).

Los productores de palma aceitera establecen estos cultivos en distintos órdenes de suelos. Esto en ocasiones muestran buenos rendimientos ( $>25$ ton/ha/año) y otros bajos ( 20 ton/ha/año) según características específicas del sitio y la implementación de buenas prácticas en el manejo agronómico y del contenido hídrico del suelo(Zakaria 1998). Sin embargo, la palma aceitera ha demostrado su mayor potencial en suelos fértiles, que aporten un buen contenido de materia orgánica y mantengan características físicas y químicas idóneas para el desarrollo del cultivo (Melado 2008). En la actualidad algunos productores utilizan ultisoles para el cultivo de la palma deaceite, sin embargo, estos suelos son muy meteorizados, presentan bajos contenidos de bases y una acidez importante causada por $\mathrm{Al}$ y Fe intercambiable en el suelo (Alvarado et al. 2014) causando bajos rendimientos, ante la ausencia de prácticas agronómicas para el mejoramiento de la fertilidad del suelo. Debido a esta situación,estas unidades edáficasse catalogan poco fértiles para la agricultura y por consecuente se debe trabajar con prácticas agronómicas diferenciadas, entre estas: las aplicaciones de distintas enmiendas químicas y orgánicas (Castro y Munévar 2013).

Es bien conocido el efecto del encalado sobre las características químicas del suelo, principalmente en la reducción de la acidez intercambiable y aumento de las bases (IPNI 1999). Por otro lado, no resta importancia las aplicaciones de otras enmiendas orgánicas, debido a que permiten optimizar las condiciones de los suelos, promoviendo unamejora en las características físicas y microbiológicas de estos (Cruz et al. 2012). Según Núñez et al. (2001) citado por Omil (2007) al utilizar cenizas que procedan de la quema de biomasa se ha convertido en una práctica comúnmente utilizada en la agricultura para incorporar una gran cantidad de nutrientes al suelo. Otros trabajos realizados por Bellote et al. (1995), Downie et al. (2009) y Guillen (2013) indicaron que el efecto de altas dosis del residuo de ceniza de las calderas, mejora significativamente propiedades físicas y químicas, entre estas: estructura, porosidad, densidad y la capacidad de retención del agua. 
El uso de residuos agroindustriales se ha utilizado en diversas investigaciones para el manejo de suelos ácidos, debido a que presentan concentraciones altas de bases, principalmente de $\mathrm{Ca}, \mathrm{Mg}$ y $\mathrm{K}$ (Ohno y Erich 1990, Etiégni et al. 1991, Clapham y Zibilske 1992, Olsson y Kellner 2002, Omil 2007, Ramírez et al. 2011, Quirantes 2015 y Escalante et al. 2016. También, estos materiales han brindado reducciones en los contenidos de acidez intercambiable y aumento del pH en los suelos. A esto se suma que la mineralización de estos materiales han ayudado en el incremento de los contenidos de macro y micro nutrimentos, aumentando el intercambio catiónico de los suelos y mejorando la nutrición en los cultivos (Lerner y Utzinger 1986, Ohno y Erich 1990, Campbell 1990, Bellote et al. 1995, Someshwar 1996; Vance 1996; Korpilathi et al. 1998; Väätäinen et al. 2000, Solla-Gullón et al. 2004, Torres et al. 2004, Othman et al. 2005, Merino et al. 2006, Odeoluwa y Adeoye 2008, Posso 2010, Guillén 2013, IICA 2016 y Madrigal et al. 2018).

Las raíces presentan un efecto positivo con las adiciones de materiales orgánicos al suelo. Es evidente el resultado que tiene la dinámica de las raíces en el tiempo, en cuanto a su distribución y salud, los efectos se han visto positivos de una forma integral según al sitio, es decir, con su relación en la fertilidad, parámetros físicos y microbiológicos en el suelo (Albertazzi et al. 2009 y Galindo y Romero 2012). Otras investigaciones realizadas por Hartley (1983) y Cristancho et al. (2007) han demostrado que en E. guineensis existen cuatro tipos de raíces, entre estas: primarias, secundarias, terciarias y cuaternarias. Los mismos autores exhiben que las raíces mostraron un tropismo positivo hacia las mejores condiciones de abastecimiento de agua, nutrimentos y materiales vegetales en descomposición. Al existir una mejor condición presente en el medio provoca que el sistema radical, principalmente en raíces finas (terciarias y cuaternarias) produzca un incremento del crecimiento (Jourdan y Rey 1997 y Albertazzi et al. 2009). Algunos investigadores indicaron que este tipo de crecimiento es catalogado en "forma de parches" (Arnone 1997, Charlton 1997). Esto se da porque las raíces absorbentes mostraron un comportamiento temporal cuando se encuentra en zonas ricas con nutrimentos en comparación a sitios en ausencia de estos (Arnone 1997, Charlton 1997 y Albertazzi 2009). Otros trabajos desarrollados por Madrigal et al. (2018) y Garbanzo et al. 2017 demostraron que las mezclas de diferentes residuos industriales (fibra, lodo y ceniza) con el suelo, presentaron un incremento en la densidad longitudinal de las raíces al incrementar las proporciones de materiales orgánicos en mezclas con suelo.

Las mejoras en las características fisicoquímicas y microbiológicas de los suelos presentan un efecto directo sobre el crecimiento y salud de las plantas. Algunos estudios han demostrado que muchos de 
estos beneficios se pueden evaluar en función del comportamiento fisiológico del cultivo (Bidwell 1993; Lallana 2004 y Romero et al. 2007). Uno de estos comportamientos es la actividad de la conductancia estomática, lo cual se encuentra estrechamente relacionada con el estado hídrico de las plantas (Torres 2013). Éste a su vez está en función del ciclo hidrológico del agua, debido a que existe una relación con el grado de apertura y cierre de las estomas según las condiciones climáticas y la dinámica hidrológica del suelo (Romero et al. 2007; y Ojeda 2015). Los estomas tienen una función esencial en el mantenimiento de la homeostasis de la planta, ya que regulan la transpiración y el ingreso de $\mathrm{CO}_{2}$ requerido en la fotosíntesis (Sánchez y Aguirreolea 2000; Chaimsohn et al. 2008 y López 2014). Algunos trabajos han demostrado que las palmas aceiteras se ven afectadas por condiciones climáticas extremas, es decir, en el caso de temperaturas elevadas se produce el cierre de estomas y en consecuencia se disminuye la fotosíntesis (Breure 2003, Corley y Tinker 2003, Raygada 2005, Melado 2008). Este comportamiento se observa en general en las plantas, ya que manejan el cierre y apertura de los estomas como un mecanismo de respuesta ante el incremento de la temperatura foliar, garantizando la turgencia y el mantenimiento del metabolismo celular, siendo el cierre de los estomas el factor que más limita directamente en la fotosíntesis de las plantas (Rees 1961, Corley 1982, Burgos et al. 1998, Melado 2008, Rojas 2015, Dell’ Amico y Morales 2017). Se ha demostrado que la conductancia estomática en las plantas presenta tasas variadas durante el día, es decir, en términos generales presentan mayores valores en horas de la mañana que en horas de la tarde, haciendo que la actividad fotosintética sea variable (Hartley 1988, Cayón 1998, Corley y Tinker 2009, Rivera 2009, Peláez et al. 2010, Reyes 2012, Rivera et al. 2013, López 2014, Rojas 2015). En síntesis, esta investigación busca analizar el efecto de la incorporación de ceniza agroindustrial de la planta extractora de aceite sobre la acidez intercambiable y la conductancia estomática en ultisoles sembrados con palma aceitera.

\section{Materiales y métodos}

Esta investigación fue desarrollada en una plantación de palma aceitera ubicada en Volcán, Buenos Aires, Puntarenas. La siembra se encuentra ubicada en las coordenadas geográficas N 09¹3'50.88" y W 83²8'57.49", a una altura de 498 msnm.

La plantación utilizada para la investigación tenía palmas adultas de ZPM años de edad y de acuerdo con las con las condiciones edáficas de la zona, el suelo se clasifica como un ultisol. Se procedió a ubicar una parcela en un sector con características de pendiente y desarrollo del cultivo homogéneas - A este sector se le realizó un análisis químico de suelos con el fin de conocer los contenidos 
nutricionales del sitio (Cuadro 1). Se utilizó una solución extractora Olsen modificado y se analizaron las siguientes variables:

- Macronutrimentos: $\mathrm{K}, \mathrm{Ca}, \mathrm{Mg}, \mathrm{P}$

- Micronutrimentos: $\mathrm{Cu}, \mathrm{Zn}, \mathrm{Mn}, \mathrm{Fe}$

- $\mathrm{pH}$ y acidez intercambiable

De la misma manera, se trasladó el residuo industrial de ceniza al sitio, este es un subproducto de las calderas utilizadas en el proceso de extracción de aceite de palma. Una vez ubicada en el sitio se procedió a realizar un análisis del contenido químico total al material, con el fin de conocer su composición química (Cuadro 2). Se implementó un análisis por medio de la técnica de digestión total y se analizaron las siguientes variables:

- Macronutrimentos: N, P, Ca, Mg, K y S

- Micronutrimentos: $\mathrm{Fe}, \mathrm{Cu}, \mathrm{Zn}, \mathrm{Mn}, \mathrm{B}$

- Porcentaje de humedad

- $\mathrm{pH}$ (agua)

- Conductividad eléctrica

- Relación $\mathrm{C} / \mathrm{N}$

Cuadro 1. Contenido de nutrientes extraíces, pH y acidez intercambiable; en el suelo de la plantación de palma aceitera usada para el desarrollo de la investigación, Buenas Aires, Puntarenas, 2019.

\begin{tabular}{ccccccccccc}
\hline & $\mathbf{p H}$ & \multicolumn{4}{c}{$\mathbf{c m o l}(+) / \mathbf{L}$} & $\mathbf{m g} / \mathbf{L}$ \\
\hline & $\mathbf{H}_{\mathbf{2}} \mathbf{O}$ & Acidez & $\mathbf{K}$ & $\mathbf{C a}$ & $\mathbf{M g}$ & $\mathbf{P}$ & $\mathbf{C u}$ & $\mathbf{Z n}$ & $\mathbf{M n}$ & $\mathbf{F e}$ \\
Nivel Crítico & 5,6 & 0.5 & 0,2 & 4 & 1 & 10 & 2 & 2 & 5 & 10 \\
Media & 4,58 & 1,73 & 0,01 & 0,37 & 0,09 & 6,80 & 4,33 & 1,43 & 2,00 & 249,66 \\
\hline
\end{tabular}

*Solución extractora Olsen modificado, Laboratorio de Suelos, Plantas, Aguas y Abonos Orgánicos, Instituto Nacional de Innovación y Transferencia en Tecnología Agropecuaria, Ministerio de Agricultura y Ganadería.

Cuadro 2. Contenido de nutrimentos totales en el residuo industrial de ceniza usada para la implementación delos tratamientos experimentales, Buenas Aires, Puntarenas, 2019.

\begin{tabular}{cccccccccccccccc}
\hline & \multicolumn{4}{c}{ \% } & masa & \multicolumn{1}{c}{$\mathbf{m g} / \mathbf{k g}$} & & \% & $\mathbf{H}_{2} \mathbf{O}$ & $\mathbf{m S} / \mathbf{c m}$ & \% & Relación \\
\hline $\mathbf{N}$ & $\mathbf{P}$ & $\mathbf{C a}$ & $\mathbf{M g}$ & $\mathbf{K}$ & $\mathbf{S}$ & $\mathbf{F e}$ & $\mathbf{C u}$ & $\mathbf{Z n}$ & $\mathbf{M n}$ & $\mathbf{B}$ & $\mathbf{H U M}$ & $\mathbf{p H}$ & $\mathbf{C E}$ & $\mathbf{C}$ & $\mathbf{C} / \mathbf{N}$ \\
0,42 & 1,46 & 3,29 & 1,30 & 3,12 & 0,09 & 10008 & 166 & 53 & 313 & 28 & 44 & 10,7 & 5,1 & 29,72 & 70,5 \\
\hline
\end{tabular}

* Laboratorio Suelos y Foliares, Centro de Investigaciones Agronómicas, Universidad de Costa Rica. 
Para el establecimiento de los tratamientos se usaron tres dosis crecientes de ceniza, aplicadas una sola vez, estas fueron: $5000 \mathrm{~kg} \mathrm{ha}^{-1}, 10000 \mathrm{~kg} \mathrm{ha}^{-1}$ y $15000 \mathrm{~kg} \mathrm{ha}^{-1}$ (Cuadro 3). Para la dosificación homogénea de las cenizas se calculó la dosis a utilizar por planta, partiendo de una densidad de siembra de 143 plantas/ha. Una vez obtenido el peso por planta se utilizó una báscula de piso digital con el fin de cuantificar la cantidad correcta de ceniza para agregar por planta. La ceniza se adicionó en la rodaja de la planta, esta tenía un área de 19,6 m². Este se realizó para todos los tratamientos y se estableció un testigo absoluto sin la adición de la ceniza.

Cuadro 3. Tratamientos utilizados para la evaluación de ceniza industrial sobre la acidez intercambiable y conductancia estomática en palma aceitera, Buenos Aires, Puntarenas, 2019.

\begin{tabular}{ccc}
\hline Tratamiento & Asignatura & $\begin{array}{c}\text { Dosis Kg ha } \\
\mathbf{1}\end{array}$ \\
\hline T1 & C5 & 5000 \\
T2 & C10 & 10000 \\
T3 & C15 & 15000 \\
T4 & Testigo & 0 \\
\hline
\end{tabular}

\section{Variables evaluadas}

Las variables se evaluaron a los 90 y 180 días después de la aplicación de la ceniza, con el fin de determinar su efecto en el tiempo. Se midió la conductancia estomática con un autoporómetro (modelo Li-Cor 1600, Li-Cor Biosciences, Lincoln, NE) el cual estaba equipado con un sensor de radiación fotosintéticamente activa $\left(\mu \mathrm{mol}\right.$ fotones $\left.\mathrm{m}^{-2} \mathrm{~s}^{-1}\right)$. Este equipo permitió caracterizar el microambiente lumínico de los foliolos de la sección medial de la hoja $\mathrm{N}^{\circ} 9$ y 17 , midiendo, además de la conductancia estomática,la apertura estomática $\left(\mathrm{mmol} \mathrm{m}^{-2} \mathrm{~s}^{-1}\right)$ y la temperatura de la hoja $\left({ }^{\circ} \mathrm{C}\right)$. Las mediciones se realizaron entre las 8:00 a.m. a 11:00 a.m. y de 1:00 p.m. a 4:00 p.m.

Así mismo, se tomaron dos muestras de raíces por palma en cada repetición, para esto se utilizó un cilindro de $200 \mathrm{~cm}^{3}$, a una distancia de 0,5 metros de la base del tronco (rodaja) y a una profundidad de $15 \mathrm{~cm}$. Las muestras se lavaron con agua para separar el suelo de las raíces, para lo que se utilizó un tamiz de 0,5 mm. Posterior a su limpieza, se secaron a temperatura ambiente y se colocaron en bandejas acrílicas transparentes de $20 \mathrm{~cm}$ x $25 \mathrm{~cm}$ x $2 \mathrm{~cm}$. Para cada muestra de raíces se digitalizó 
una imagen con un escáner marca Epson V700 modelo "Perfection" con una resolución de 600 dpi. Para esto se utilizó una lámina de color azul en el fondo con el fin de mejorar el contraste de la imagen y así reducir la formación de sombras. Las imágenes fueron analizadas con el paquete informático WinRhizo ${ }^{\circledR}$ Pro, empleando una calibración específica para palma aceitera y permitiendo la realización de mediciones de las raíces en términos de su morfología y color. De acuerdo con el diámetro, las raíces se cuantificaron como: primarias $(>5 \mathrm{~mm})$, secundarias $(1,5 \mathrm{a} 5 \mathrm{~mm})$, terciarias $(0,5$ a $1,5 \mathrm{~mm})$ y cuaternarias $(<0,5 \mathrm{~mm})$. La medición de la longitud total de las raíces según la categoría, se dividió entre el volumen del cilindro conocido para obtener la densidad de raíces, definida en términos de $\mathrm{cm} / \mathrm{cm}^{3}$, todo esto según la metodología empleada por Gutiérrez et al. (2014).

Se analizó el comportamiento del contenido químicode nutrimentos en el suelo, a los 90 y 180 dda los residuos industriales. Para esto se recolectaron submuestras por planta para generar una muestra compuesta por cada repetición de los tratamientos. Estas fueron llevadas al Laboratorio de Suelos, Plantas, Aguas y Abonos Orgánicos del Instituto Nacional de Innovación y Transferencia en Tecnología Agropecuaria (INTA) para determinar el pH en agua, acidez intercambiable, $\mathrm{Ca}, \mathrm{Mg}, \mathrm{K}$, $\mathrm{P}, \mathrm{Fe}, \mathrm{Zn}, \mathrm{Mn}, \mathrm{Cu}, \mathrm{y} \mathrm{Al}$ mediante extracciones con KCL-Olsen Modificado.

\section{Análisis estadístico}

Se generó una base de datos con las variables: propiedades fisicoquímicas del suelo, las variables de crecimiento, desarrollo de raíz y la conductancia estomática. Se procedió a realizar un promedio de las observaciones de campo para la tabulación y graficación de los resultados. Así mismo se utilizó la base de datos general para realizarle pruebas de supuestos básicos de homogeneidad y normalidad de los datos, así mismo se procedió a realizar un análisis de varianza. Posteriormente se le aplicó la prueba de Tukey $(\alpha=0,05)$ con el fin de comparar todos los tratamientos entre sí e identificar separaciones ente las medias de cada variable. Todos estos datos se realizaron por medio del software estadístico Infostat.

\section{Resultados}

Efecto de las dosis crecientes en el suelo a los 90 y 180 días después de aplicados los tratamientos Se encontró una tendencia en el aumento del pH y una disminución de la acidez intercambiable del suelo, con respecto al tratamiento testigo, a los90 dda del residuo industrial (Cuadro 4). Al evaluar la concentraciones de nutrimentos en el suelo, el $\mathrm{P}$ mostró un aumento $(7,53 \mathrm{cmol}(+) / \mathrm{L})$ significativo 
$(\alpha=0,05)$ usando $10000 \mathrm{~kg} \mathrm{ha}^{-1}$ de ceniza a los 90 dda (Cuadro 4). Para las demás variables (K, Ca, $\mathrm{Mg}, \mathrm{Cu}, \mathrm{Zn}, \mathrm{Mn}, \mathrm{Fe}$ ) no se encontraron diferencias estadísticas significativas en comparación de todos los tratamientos. Se evidenció una ligera disminución en la la acidez intercambiable y un aumento del pH con respecto a la media (Cuadro 1). El nutriente $\mathrm{K}$ mostro un aumento en los tratamientos C5, C10 y C15 en relación al testigo $(0,03 \mathrm{cmol}(+) / \mathrm{L})$. Se manifestó una tendencia en la disminución de los contenidos de Ca y el Mg en el suelo de los tratamientos en comparación a los valores que se presentaron en el Cuadro 1.

Cuadro 4. Concentración de nutrimentos, $\mathrm{pH}$ y acidez intercambiable del suelo de los tratamientos de la investigación a los 90 y 180 días después de la aplicación (dda) de la ceniza en Buenos Aires, Puntarenas, 2019.

\begin{tabular}{|c|c|c|c|c|c|c|c|c|c|c|}
\hline \multirow{3}{*}{ Tratamientos } & \multicolumn{10}{|c|}{90 dda } \\
\hline & \multirow[t]{2}{*}{ pH } & \multicolumn{4}{|c|}{$\operatorname{cmol}(+) / \mathbf{L}$} & \multicolumn{5}{|c|}{$\mathrm{mg} / \mathrm{L}$} \\
\hline & & Acidez & K & $\mathbf{C a}$ & Mg & $\mathbf{P}$ & $\mathbf{C u}$ & $\mathbf{Z n}$ & Mn & $\mathrm{Fe}$ \\
\hline C 5 & 4,75 & 1,28 & 0,05 & 0,28 & 0,07 & $3,83^{\mathrm{ab}}$ & 3,75 & 1,13 & 1,25 & 284,75 \\
\hline C 10 & 4,74 & 1,35 & 0,04 & 0,25 & 0,07 & $7,53^{\mathrm{b}}$ & 3,75 & 0,68 & 1,00 & 284,75 \\
\hline C 15 & 4,73 & 1,34 & 0,05 & 0,21 & 0,08 & $4,05^{\mathrm{ab}}$ & 3,75 & 0,75 & 1,00 & 292,00 \\
\hline \multirow{2}{*}{ Testigo } & 4,70 & 1,34 & 0,03 & 0,21 & 0,06 & $2,40^{\mathrm{a}}$ & 4,25 & 0,65 & 1,00 & 283,25 \\
\hline & \multicolumn{10}{|c|}{180 dda } \\
\hline C 5 & 4,81 & 1,25 & $0,04^{\mathrm{a}}$ & 0,32 & 0,09 & 3,83 & 2,75 & 0,55 & 0,75 & 263,25 \\
\hline C 10 & 4,86 & 1,20 & $0,05^{\mathrm{a}}$ & 0,28 & 0,08 & 2,35 & 3,25 & 0,43 & 1,00 & 277,50 \\
\hline C 15 & 4,88 & 1,32 & $0,10^{\mathrm{b}}$ & 0,35 & 0,16 & 6,58 & 3,50 & 1,05 & 1,00 & 287,50 \\
\hline Testigo & 4,79 & 1,38 & $0,06^{\mathrm{ab}}$ & 0,29 & 0,08 & 4,35 & 3,25 & 0,78 & 0,75 & 281,75 \\
\hline
\end{tabular}

A los 180 dds se encontró que el $\mathrm{K}$ mostró una diferencia significativa $(\alpha=0,05)$ en los tratamientos C5 $(0,04 \mathrm{cmol}(+) / \mathrm{L})$ y C10 $(0,06 \mathrm{cmol}(+) / \mathrm{L})$, con respecto al testigo(Cuadro 4). Sin embargo este efecto no se encontró para el tratamiento $\mathrm{C} 15(0,10 \mathrm{cmol}(+) / \mathrm{L})$. El pH aumentó levemente en los tratamientos y la acidez disminuyó con respecto al testigo???, pero no se manifestó una diferencia significativa. Así mismo, se observó un aumento del Mg en el tratamiento C15 (0,16 cmol(+)/L) a los 180 dda con respectoal testigo???. Los elementos Ca, P, Cu, Zn y Mn se mantuvieron por debajo de los resultados que se obtuvieron en la media, a excepción del Fe que presentó un mayor valor. La acidez intercambiable manifestó una disminución no significativa en los tratamientos C5, C10 y C15 en los cuales se les aplicó ceniza en comparación al testigo (1,38 cmol(+)/L). Por último, se encontró que los tratamientos C5 y C15 mostraron mayores concentraciones de Ca y Mg en cuanto al testigo a los 180 dda. 
Crecimiento y desarrollo de la raíz a partir de las dosis de ceniza aplicadas en los distintos tratamientos

El desarrollo del sistema radical no mostró limitaciones en el crecimiento bajo la influencia de los tratamientos (Cuadro 5). La densidad de raíz a los 90 días de la aplicación (dda) no mostró diferencia estadística significativa $(\alpha=0,05)$ en ninguno de los tratamientos, este efecto fue similar para la evaluación a los 180 dda. Al analizar las tendencias de crecimiento y desarrollo de las raíces, se encontró que el tratamiento C10 a los 90 dda expresó mayores tendencias de crecimiento en la densidad de raíz $\left(29,10 \mathrm{~cm} / \mathrm{cm}^{3}\right)$ que los demás tratamientos, lo que podría indicar una mayor respuesta en esta variable ante la aplicación de $10000 \mathrm{~kg} \mathrm{ha}^{1}$ de ceniza. De acuerdo con los resultados que se manifestaron a los 180 dda, se presentó unaumento de la densidad de raíz en todos los tratamientos? en comparación a los 90 dda. En ambas fechas de medición (90 y 180 dda) se presentó en orden creciente, el siguiente promedio de densidad de raíces para cada tratamiento : C10 (2,54 $\left.\mathrm{cm} / \mathrm{cm}^{3}\right)$, testigo $\left(5,49 \mathrm{~cm} / \mathrm{cm}^{3}\right), \mathrm{C} 15\left(11,32 \mathrm{~cm} / \mathrm{cm}^{3}\right)$ y C5 $\left(11,95 \mathrm{~cm} / \mathrm{cm}^{3}\right)$.

Al adicionar residuos de ceniza industrial se encontró un aumento en el crecimiento de raíces terciarias y cuaternarias (Cuadro 5). A los 90 dda el tratamiento C10 mostro un mayor crecimiento, no significativo, en las raíces terciarias $\left(20,23 \mathrm{~cm} / \mathrm{cm}^{3}\right)$ y cuaternarias $\left(7,37 \mathrm{~cm} / \mathrm{cm}^{3}\right)$ con respecto al testigo $\left(\mathrm{xxx} \mathrm{cm} / \mathrm{cm}^{3}\right)$. A los $180 \mathrm{dda}$, los tratamientos $\mathrm{C} 5 \mathrm{y}$ C15 mantuvieron un crecimiento superior en las raíces secundarias, terciarias y cuaternarias $\left(1,97 \mathrm{~cm} / \mathrm{cm}^{3}, 22,80 \mathrm{~cm} / \mathrm{cm}^{3}, 8,54 \mathrm{~cm} / \mathrm{cm}^{3}\right)$ en relación con el testigo $\left(\mathrm{xxx} \mathrm{cm} / \mathrm{cm}^{3}\right)$.

Cuadro 5. Comportamiento en la densidad de raíces a los 90 y 180 días después de la aplicación de ceniza industrial en palma aceitera en Buenos Aires, Puntarenas, 2019.

\begin{tabular}{cccccccccc}
\hline \multirow{2}{*}{ Tratamiento } & \multicolumn{4}{c}{ 90 dda } & \multicolumn{5}{c}{ 180 dda } \\
\cline { 2 - 9 } & $\mathbf{C ~ 5}$ & $\mathbf{C ~ 1 0}$ & $\mathbf{C ~ 1 5}$ & Testigo & $\mathbf{C ~ 5}$ & $\mathbf{C ~ 1 0}$ & $\mathbf{C ~ 1 5}$ & Testigo \\
\cline { 2 - 9 } & \multicolumn{4}{c}{$\left(\mathbf{c m} / \mathbf{c m}^{\mathbf{3}}\right)$} \\
\hline Raíz Primaria & 0,01 & 0,01 & 0,01 & 0,01 & 0,01 & 0,01 & 0,03 & 0,03 \\
Raíz Secundaria & 1,76 & 1,47 & 1,41 & 1,49 & 2,35 & 1,56 & 2,10 & 1,97 \\
Raíz Terciaria & 17,80 & 20,23 & 18,62 & 19,23 & 26,49 & 21,94 & 26,62 & 22,80 \\
Raíz Cuaternaria & 6,70 & 7,37 & 6,24 & 7,12 & 9,36 & 8,11 & 8,83 & 8,54 \\
Densidad total de Raíz & 26,28 & 29,10 & 26,29 & 27,87 & 38,23 & 31,64 & 37,61 & 33,36 \\
\hline
\end{tabular}


La densidad de raíz mostró un comportamiento no significativo del muestreo que se realizó a los 90 y 180 dda del residuo industrial de ceniza, pero si reveló una tendencia con respecto a los datos obtenidos (Figura 1). A los 90 dda el tratamiento C10 $\left(29,10 \mathrm{~cm} / \mathrm{cm}^{3}\right)$ expresó mayor densidad de raíz en comparación al testigo $\left(27,87 \mathrm{~cm} / \mathrm{cm}^{3}\right)$. En los 180 dda se revelo una superioridad en la densidad de raíz de los tratamientos C5 y C15 con relación al testigo $\left(33,36 \mathrm{~cm} / \mathrm{cm}^{3}\right)$ mostrando la influencia positiva del residuo industrial en el crecimiento radicular.

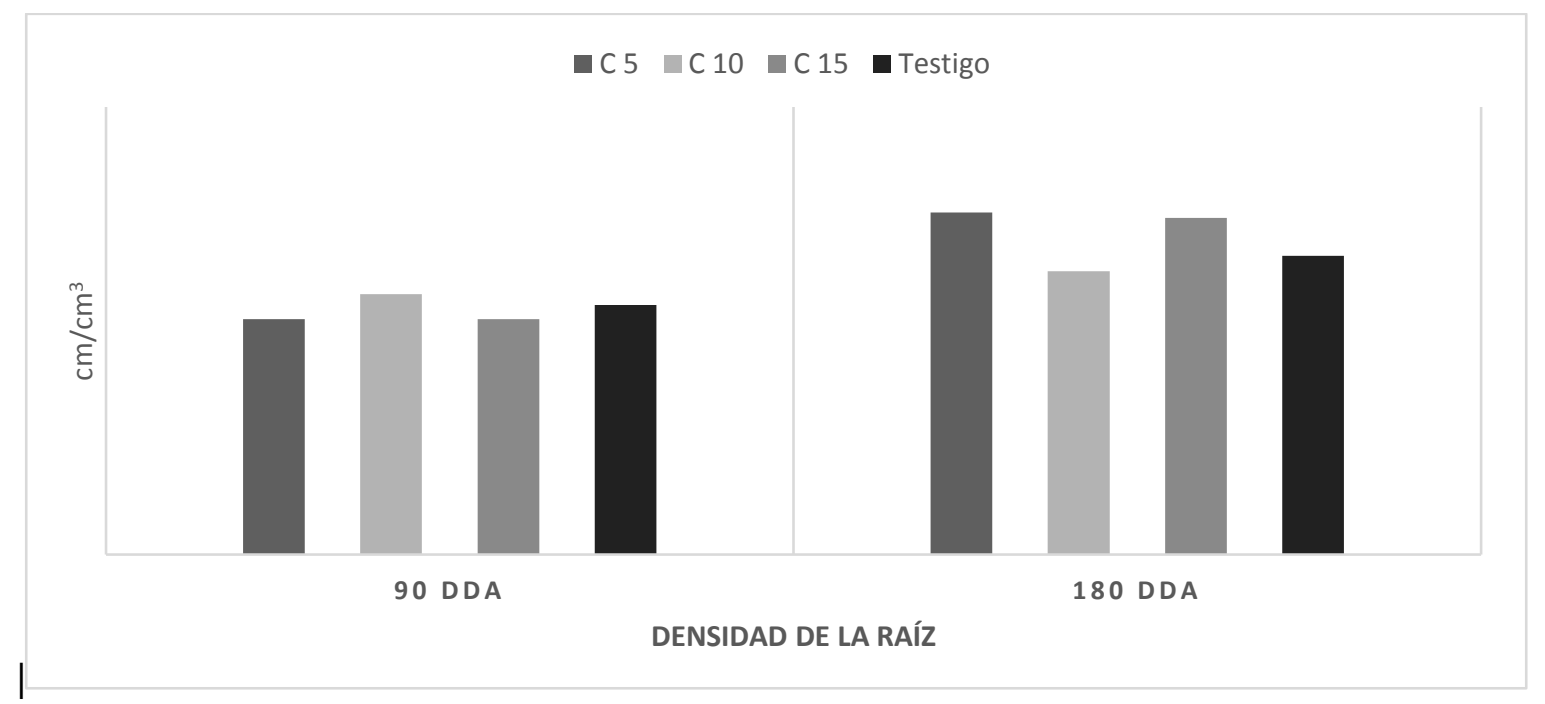

Figura 1. Efecto de la adición de distintas concentraciones de residuos de ceniza sobre la densidad de raíces en palma aceitera a los 90 y 180 después de aplicado en las plantas en Buenos Aires, Puntarenas, 2019.

\section{Variación de la conductancia estomática y la temperatura a los 90 y 180 días de aplicación}

La conductancia estomática antes del mediodía (AM) en la hoja $\mathrm{N}^{\circ} 9$ mostró diferencias significativas $(\alpha=0,05)$ en los tratamientos C5, C10 y C15 con respeto al testigo $\left(243,93 \mathrm{mmol} \mathrm{m}^{-2} \mathrm{~s}^{-1}\right)$ (Cuadro 6 y Figura 2). La hoja $\mathrm{N}^{\circ} 17$ reveló diferencia significativa en la temperatura del tratamiento $\mathrm{C} 5$ (28,85 $\left.{ }^{\circ} \mathrm{C}\right)$ con respecto al testigo $\left(29,78{ }^{\circ} \mathrm{C}\right)$. 
Cuadro 6. Variación de la conductancia estomática y la temperatura en las plantas de palma aceitera a los 90 y 180 días después de aplicación de la ceniza en Buenos aires, Puntarenas, 2019.

\begin{tabular}{|c|c|c|c|c|c|c|c|c|}
\hline \multirow[b]{3}{*}{ Tratamiento } & \multicolumn{4}{|c|}{$\mathbf{A M}$} & \multicolumn{4}{|c|}{ PM } \\
\hline & \multicolumn{2}{|c|}{ Hoja 9} & \multicolumn{2}{|c|}{ Hoja 17} & \multicolumn{2}{|c|}{ Hoja 9} & \multicolumn{2}{|c|}{ Hoja 17} \\
\hline & 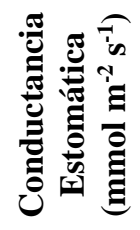 & 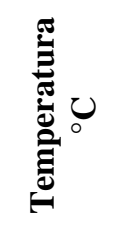 & 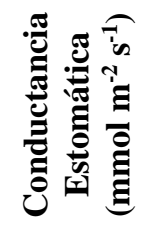 & 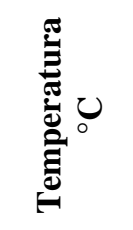 & 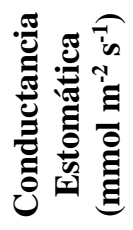 & 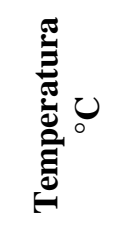 & 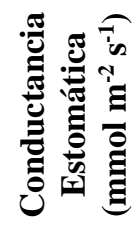 & 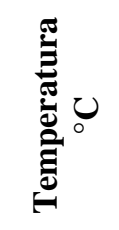 \\
\hline \multicolumn{9}{|c|}{90 dda } \\
\hline C 5 & $524,40^{\mathrm{b}}$ & 28,73 & 514,18 & $28,85^{\mathrm{a}}$ & 208,95 & 31,50 & 178,60 & 31,53 \\
\hline C 10 & $436,90^{b}$ & 29,08 & 522,73 & $29,10^{\mathrm{ab}}$ & 169,10 & 31,40 & 143,53 & 31,35 \\
\hline C 15 & $558,93^{b}$ & 29,70 & 452,30 & $29,45^{a b}$ & 160,95 & 31,28 & 84,48 & 31,18 \\
\hline Testigo & $243,93^{a}$ & 29,70 & 250,18 & $29,78^{\mathrm{b}}$ & 137,28 & 31,10 & 84,65 & 31,15 \\
\hline \multicolumn{9}{|c|}{180 dda } \\
\hline C 5 & 597,00 & $27,63^{\mathrm{a}}$ & $621,08^{b}$ & $27,75^{\mathrm{a}}$ & 174,23 & $32,35^{\mathrm{a}}$ & 269,15 & $32,60^{\mathrm{a}}$ \\
\hline C 10 & 679,43 & $28,95^{\mathrm{b}}$ & $622,90^{\mathrm{b}}$ & $28,95^{\mathrm{b}}$ & 127,98 & $33,25^{a b}$ & 288,33 & $33,38^{a b}$ \\
\hline C 15 & 604,50 & $29,95^{c}$ & $293,20^{\mathrm{a}}$ & $30,05^{\mathrm{c}}$ & 443,30 & $33,35^{\mathrm{ab}}$ & 104,10 & $33,20^{a b}$ \\
\hline Testigo & 465,65 & $31,03^{\mathrm{d}}$ & $406,60^{a b}$ & $31,08^{\mathrm{d}}$ & 96,03 & $34,15^{\mathrm{b}}$ & 128,00 & $34,20^{\mathrm{b}}$ \\
\hline
\end{tabular}

Medias seguidas por una misma letra no representan diferencias significativas según prueba de Tukey $(\alpha=0,05)$

La mediciones que se efectuarondespués del mediodía (PM) no manifestaron diferencias significativas en la hoja $\mathrm{N}^{\circ} 9$ y 17 en la conductancia estomática y la temperatura en ninguno de los tratamientos (Cuadro 6). Sin embargo, la conductancia estomática de la hoja $\mathrm{N}^{\circ} 9$ fue mayor en los tratamientos C5, C10 y C15 en comparación al testigo $\left(137,28 \mathrm{mmol} \mathrm{m}^{-2} \mathrm{~s}^{-1}\right)$. A su vez, La hoja $\mathrm{N}^{\circ} 17$ mostró una mayor conductancia estomática en los tratamientos $\mathrm{C} 5\left(178,60 \mathrm{mmol} \mathrm{m}^{-2} \mathrm{~s}^{-1}\right)$ y C10 $\left(143,53 \mathrm{mmol} \mathrm{m}^{-2} \mathrm{~s}^{-1}\right)$ con respecto al testigo $\left(84,65 \mathrm{mmol} \mathrm{m}^{-2} \mathrm{~s}^{-1}\right)$. 


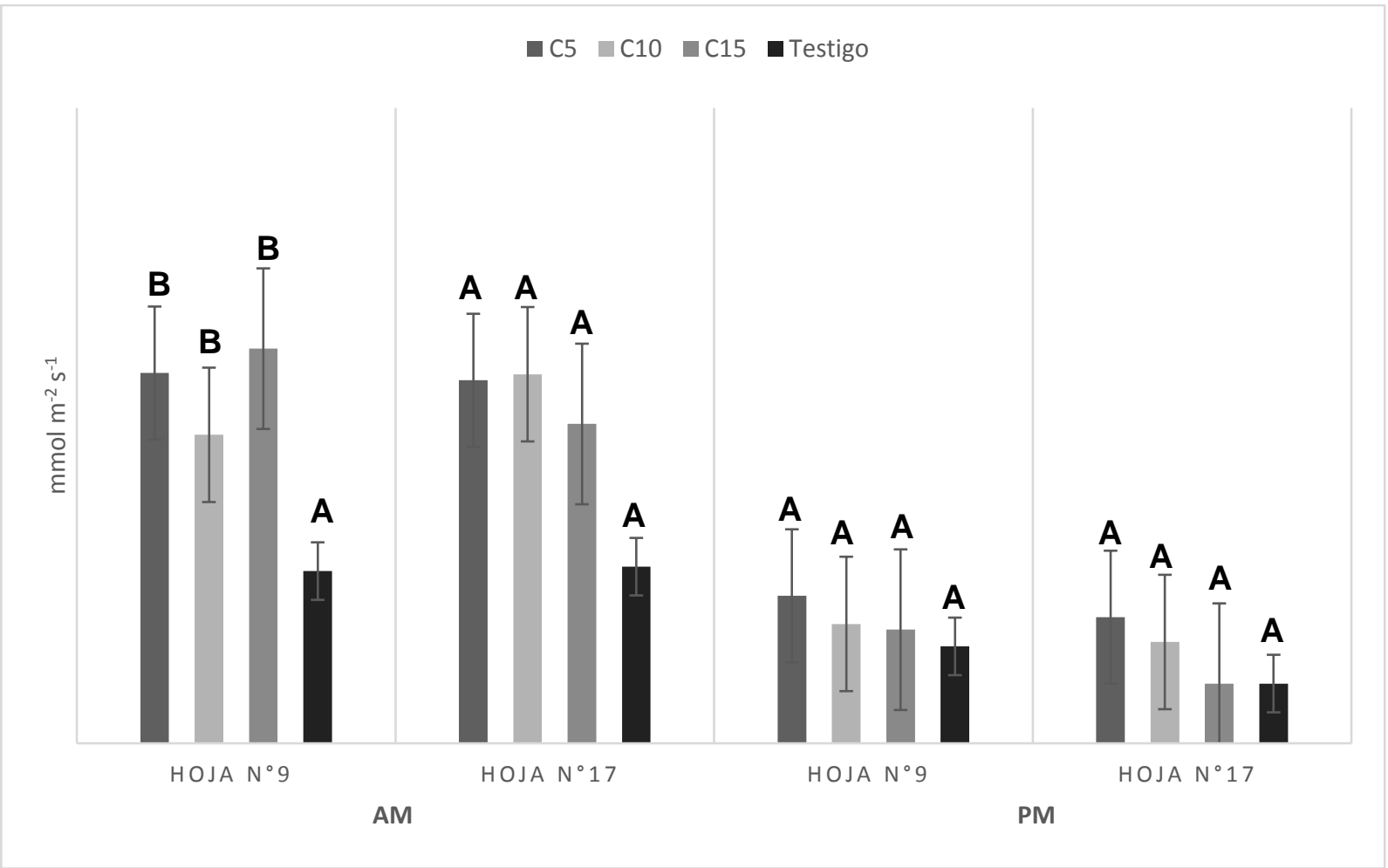

Figura 2. Variación de la conductancia estomática en plantas de palma aceitera a los 90 días después de aplicación de la ceniza en Buenos aires, Puntarenas, 2019.

La hoja $\mathrm{N}^{\circ} 9$ antes del mediodía (AM) no mostró diferencias significativas en la conductancia estomática a los 180 dda (Cuadro 6 y Figura 3). Sin embargo, los resultados de los tratamientos indicaron ser superiores en relación con el testigo $\left(465,65 \mathrm{mmol} \mathrm{m}^{-2} \mathrm{~s}^{-1}\right)$. La temperatura en la hoja $\mathrm{N}^{\circ} 9$ indicó diferencias estadísticas significativas $(\alpha=0,05)$ para los tratamientos $\mathrm{C} 5, \mathrm{C} 10$ y C15 con respecto al testigo $\left(31,03{ }^{\circ} \mathrm{C}\right)$. Las mediciones de conductancia estomática en la hoja $\mathrm{N}^{\circ} 17$ reveló que los tratamientos C5 $\left(621,08 \mathrm{mmol} \mathrm{m}^{-2} \mathrm{~s}^{-1}\right)$ y $\mathrm{C} 10\left(622,90 \mathrm{mmol} \mathrm{m}^{-2} \mathrm{~s}^{-1}\right)$ difieren estadísticamente del tratamiento C15 $\left(293,20 \mathrm{mmol} \mathrm{m}^{-2} \mathrm{~s}^{-1}\right)$. A su vez la temperatura en la hoja $\mathrm{N}^{\circ} 17$ expresó diferencias estadísticas significativas $(\alpha=0,05)$ en los tratamientos $\mathrm{C} 5, \mathrm{C} 10$ y $\mathrm{C} 15$ (bbbvvvfff ${ }^{\circ} \mathrm{C}$ ). con respecto al testigo $\left(31,08^{\circ} \mathrm{C}\right)$.

La conductancia estomática después del mediodía (PM) no manifestó diferencias significativas en la hoja $\mathrm{N}^{\circ} 9$ y 17 (Cuadro 6 y Figura 3). Los tratamientos C5, C10 y C15 en la hoja $\mathrm{N}^{\circ} 9$ presentaron mayor conductancia estomática que el testigo $\left(96,03 \mathrm{mmol} \mathrm{m}^{-2} \mathrm{~s}^{-1}\right)$. Así mismo, la temperatura de la hoja $\mathrm{N}^{\circ} 9$ y 17 mostró una diferencia significativa $(\alpha=0,05)$ del tratamiento $\mathrm{C} 5\left(32,35^{\circ} \mathrm{C}\right.$ y $\left.32,60{ }^{\circ} \mathrm{C}\right)$ en relación con el testigo $\left(34,15^{\circ} \mathrm{C}\right.$ y $\left.34,20^{\circ} \mathrm{C}\right)$. 
Los tratamientos C5, C10 y C15 expresaron una mayor conductancia estomática en las hojas $\mathrm{N}^{\circ} 9$ y 17 a los 90 dda en referencia al testigo (Figura 2). A los 180 dda la conductancia estomática del tratamiento testigo fue inferior únicamente en la hoja $\mathrm{N}^{\circ} 9$ en relación con los tratamientos $(\mathrm{C} 5, \mathrm{C} 10$ y C15). La Hoja $\mathrm{N}^{\circ} 17$ revelo una mayor conductancia estomática en los tratamientos C5 y C10 a los 180 dda con respecto al testigo. Por último, la temperatura fue inferior en los tratamientos C5, C10 y C15 con relación al testigo a los 180 dda (Cuadro 6).

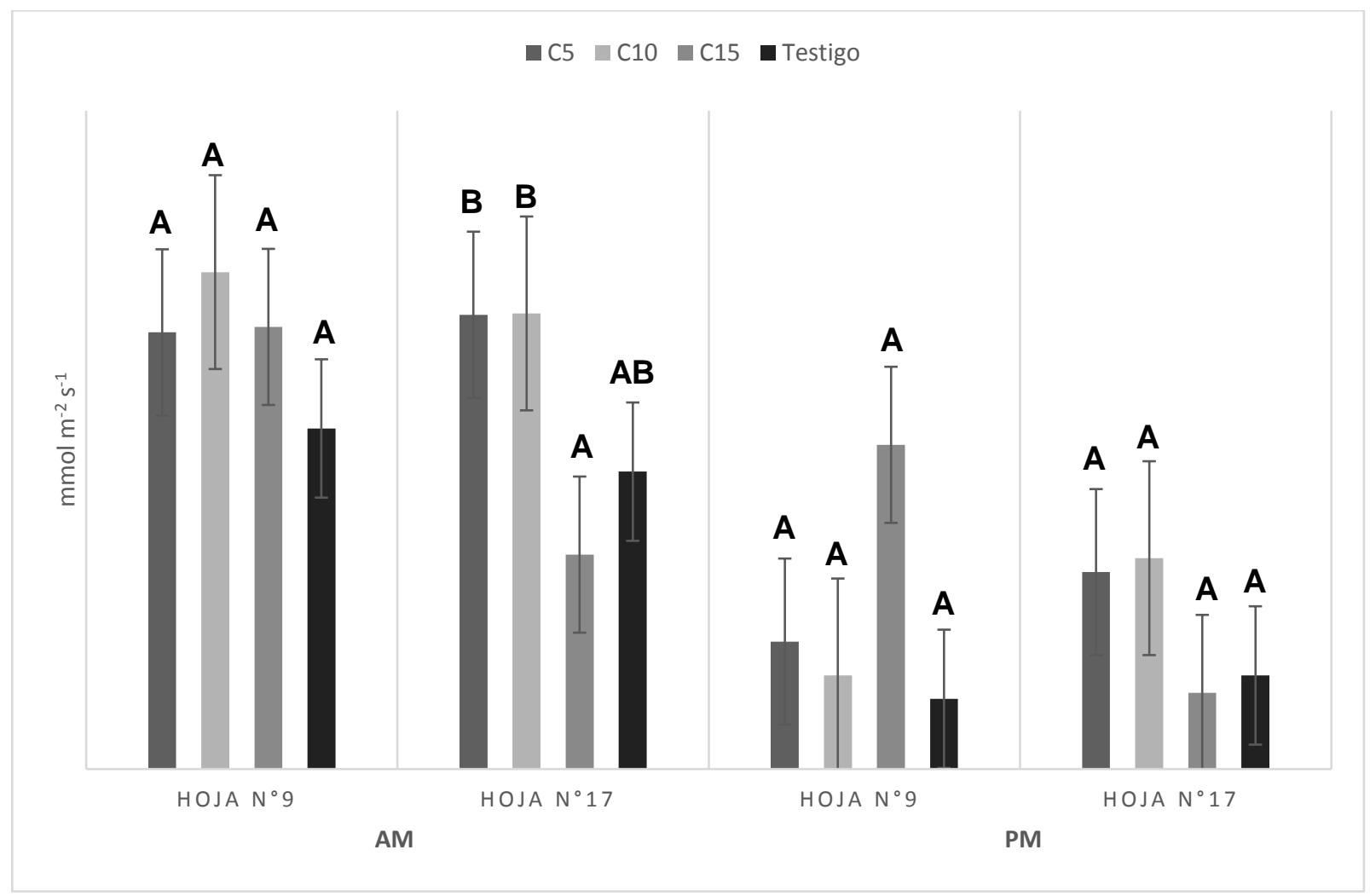

Figura 3. Variación de la conductancia estomática en plantas de palma aceitera a los 180 días después de aplicación de la ceniza en Buenos aires, Puntarenas, 2019.

\section{Discusión}

El efecto en el suelo de las dosis utilizadas mostró una tendencia ligera a incrementar el pH y disminuir la acidez intercambiable a los 90 y 180 dda, en respuesta a la adición de $5000 \mathrm{~kg} \mathrm{ha}^{-1}$ (C5); la misma respuesta ocurrió en los tratamientos de $10000 \mathrm{~kg} \mathrm{ha}^{-1}$ (C10) y $15000 \mathrm{~kg} \mathrm{ha}^{-1}$ (C15) a los 180 dda. Resultados que pudieron haberse mostrado debido a que la ceniza residual utilizada posee un efecto alcalino (pH 10,7) (Cuadro 2). Esto concuerda con lo mencionado por Ramírez et al. (2011), Guillén (2013), Quirantes (2015), Escalante et al. (2016) y IICA (2016) en donde se indicó que la ceniza mantiene una alta alcalinidad. La mayoría de los trabajos se centran en el uso de enmiendas 
orgánicas con el fin de elevar el pH de los suelos con problemas. Otras investigaciones realizadas sobre la utilización de residuos de ceniza han demostrado una correlación positiva con el incremento del $\mathrm{pH}$ y concluyeron que dicho efecto era similar al de cualquier otro material encalante por poseer alto contenido de bases (Etiégni et al. 1991; Clapham y Zibilske 1992; Olsson y Kellner 2002 y Omil 2007). Por otro lado, este mismo efecto se ha encontrado con la disminución de la acidez intercambiable. Algunas investigaciones reportan que al aplicar ceniza o materiales orgánicos tienen efecto sobre la disminución del contenido de Al intercambiable en suelo (Lerner y Utzinger 1986; Ohno y Erich 1990; Solla-Gullón et al. 2004; Merino et al. 2006; Omil 2007; Guillen 2013 y Madrigal et al. 2018).

La aplicación de residuos de cenizas en suelos ácidos de palma aceitera incremento los contenidos de $\mathrm{P}$ en el suelo. El mayor efecto e el incremento del contenido de este nutrimentonse observó a los 90 dda, debido a la incorporación de grandes cantidades del residuo industrial generaron un aporte significativo en las concentraciones de P. Bellote et al. (1995) indicó en sus trabajos que aplicaciones de altas dosis de ceniza de las calderas, mejoraron significativamente las concentraciones químicas en el suelo y entre los cuales se recalcaron los contenidos de P. Estos cambios se dan debido a que las cenizas de biomasa contienen todos los elementos necesarios para el crecimiento de la vegetación (Korpilathi et al. 1998). Torres et al. (2004), Posso (2010) y Madrigal et al. (2018) indicaron que al adicionar materiales orgánicos o residuos (compost o ceniza) estos son muy ricos en nutrimentos principalmente en $\mathrm{P}$ y coincide también con la referencia de los estudios realizados por Odeoluwa y Adeoye (2008). También Cruz et al. (2012) indicó que el uso de la materia orgánica en los suelos agrícolas mejoró considerablemente las características fisicoquímicas de estos en el tiempo.

Las concentraciones de $\mathrm{K}$ aumentaron con la aplicación de residuos de ceniza en suelos ácidos a los 180 dda. Se encontró un efecto significativo con las concentraciones de K en el suelo a los 180 dda para los tratamientos C5 y C10. Las aplicaciones en altas concentraciones de este residuo promovieron un mayor incremento de este nutrimento en la fase soluble del suelo a los 180 dda, indicando una alta disponibilidad para las plantas la y posiblemente este efecto no se observe significativamente a los $90 \mathrm{dda}$, debido a que el material necesita un tiempo superior para su mineralización. Algunos trabajos han reportado que las aplicaciones de materiales orgánicos logran aportar al suelo importantes contenidos de $\mathrm{K}$, siempre y cuando los materiales tengan concentraciones altas de este nutrimento (Campbell 1990; Someshwar 1996; Vance 1996; Torres et al. 2004 y Madrigal et al. 2018). Los residuos agroindustriales de la palma aceitera (fibra, lodos y cenizas) han mostrado efectos químicos positivos al ser aplicados al suelo y en cuanto al caso específico del residuo 
de ceniza, otros estudios indicaron que existen aportes en la concentración de N, P, K, Ca, Mg y micronutrientes (Bellote et al. 1995; Korpilathi et al. 1998; Solla-Gullón et al. 2004; Odeoluwa y Adeoye 2008 y Posso 2010, Garbanzo et al. 2017). La relación existente entre el material residual y el tiempo son factores que limitan la disponibilidad de los nutrimentos para que estos logran mineralizarse por completo (Väätäinen et al. 2000).

La aplicación de residuos de ceniza en suelos ácidos logró incrementar las raíces terciarias y cuaternarias en palma aceitera. El mayor efecto del crecimiento radical se observó a los 180 dda en los tratamientos C5 $\left(38,23 \mathrm{~cm} / \mathrm{cm}^{3}\right)$ y C15 $\left(37,61 \mathrm{~cm} / \mathrm{cm}^{3}\right)$ en relación al testigo $\left(33,36 \mathrm{~cm} / \mathrm{cm}^{3}\right)$, probablemente debido a que la mejora química del suelo logró estimular el crecimiento de estas raíces, lo cual es un efecto positivo para la nutrición del cultivo. Se sabe bien que los residuos orgánicos cumplen funciones muy importantes entre éstas: la mejora de condiciones físicas, químicas, biológicas y retención de humedad en el suelo. Esto concuerda con Jourdan y Rey (1997); Galindo y Romero (2012) y Madrigal et al. (2018) los cuales indicaron que la dinámica del tiempo en el crecimiento radical de la palma aceitera depende de factores o condiciones idóneas para el abastecimiento de nutrientes, agua y materia orgánica.

Se sabe que las raíces de la palma aceitera buscan condiciones óptimas para el desarrollo, en materia de aspectos fisicoquímicos y microbiológicos del suelo. Estas propiedades garantizan un desarrollo en el sistema radical, permitiendo que la planta pueda absorber nutrimentos y oxigeno de una manera más eficiente (Albertazzi et al. 2009, Garbanzo 2018 y Madrigal et al. 2018). Downie et al. (2009) y Guillén (2013) indicaron que la incorporación del residuo industrial de ceniza al suelo pudo ocasionar cambios en las propiedades físicas, tales como: estructuración, disminución de la densidad aparente y aumento de la porosidad. Esta última con implicaciones directas en la aireación del suelo, causando un incremento de la permeabilidad hídrica y la conductividad hidráulica, logrando disminuir los impactos por erosión.

La aplicación de residuos de ceniza agroindustrial en suelos ácidos de palma aceitera logró tener efectos en la conductancia estomática de hojas 9 y 17. Se encontró que la conductancia estomática en la hoja $\mathrm{N}^{\circ} 9$ antes del mediodía a los 90 dda incrementó significativamente para los tratamientos C5, $\mathrm{C} 10$ y C15. Es probable que este efecto sea producto de una mayor cantidad de agua y nutrimentos en el suelo. Burgos et al. (1998) encontró que las plantas que presentaron una alta humedad en el suelo mostraron mayores valores en la conductancia estomática y principalmente se observó la mejor respuesta en hojas más jóvenes (hoja $\mathrm{N}^{\circ}$ ). Así mismo, se encontró que la conductancia estomática 
en la hoja $\mathrm{N}^{\circ} 17$ antes del mediodía a los 90 dda, incrementó su valor para los tratamientos (C5, C10 y C15), lo cual se relacionó con la temperatura de la hoja, la cual incrementó para el testigo con relación al tratamiento $\mathrm{C} 5\left(28,85^{\circ} \mathrm{C}\right)$, indicando un menor intercambio gaseoso en las palmas que no se les aplicó ceniza industrial. Downie et al. (2009) y Guillén (2013) determinaron que la adicción de ceniza en los suelos incrementó positivamente la capacidad de retención de agua. La palma también tiene una adaptación al cierre y apertura de las estomas según las temperaturas de la zona, lo cual, ayuda a la planta a mantener un estado hídrico y fotosintético óptimo (Breure 2003; Corley y Tinker 2003; Raygada 2005 y Melado 2008, Dell' Amico y Morales 2017). Esto se debe a que la palma de aceite es una planta C3 y muestra una relación curvilínea con la intensidad de la luz y se ve afectada por la elevación de la temperatura foliar (Rees 1961, Corley 1982, Breure 2003, Melado 2008, Corley y Tinker 2009, citado por Peláez et al. 2010, Rojas 2015).

A los 180 dda la conductancia estomática en la hoja $\mathrm{N}^{\circ} 9$ fue mayor en los tratamientos C5, C10 y C15 en relación con el testigo $\left(465,65 \mathrm{mmol} \mathrm{m}^{-2} \mathrm{~s}^{-1}\right)$ y se relacionó con la diferencia significativa de la temperatura. Los incrementos de la temperatura en las plantas o el medio donde se encuentran estas situadas provocan el cierre de estomas disminuyendo por ende la conductancia estomática en la planta. Este aspecto se relaciona a lo indicado por algunos investigadores en donde se debe a que el aumento de la temperatura foliar puede inhibir claramente en el cierre de estomas y este es el factor que mayormente limita la actividad fotosintética de las plantas (Rees 1961; Corley 1982; Burgos et al. 1998; Pelaez et al. 2010; López 2014; Rojas 2015 y Melado 2008). La hoja N 17 mostro diferencia significativa sobre la conductancia y la temperatura entre los tratamientos (C5, C10 y C15) respecto con el testigo (406,60 $\left.\mathrm{mmol} \mathrm{m} \mathrm{m}^{-2} \mathrm{~s}^{-1}\right)$. Así mismo, el mayor comportamiento de la conductancia estomática en las hojas se da cuando la temperatura es baja y este proceso fisiológico disminuye conforme asciende la temperatura. Según Reyes (2012) y Rojas (2015) la fotosíntesis muestra un sistema bimodal manifestando los mayores valores en las horas de la 9:00 am a 11:00 am y de 3:00 pm a 5:00 pm. En otras investigaciones se indicó que el mayor comportamiento estomático es realizado entre las 9:00 am a 11:00 a.m. y su disminución se da en las horas de 12:00 pm a 3:00 p.m., principalmente por el aumento de la temperatura ambiental y la combinación de otros factores (Rivera 2009; Peláez et al. 2010; Reyes 2012; Rivera et al. 2013; López 2014; Rojas 2015; Dell’ Amico y Morales 2017).

Las mediciones efectuadas después del mediodía presentaron diferencias significativas en ambas hojas con respecto a la temperatura de los tratamientos $(\mathrm{C} 5, \mathrm{C} 10$ y $\mathrm{C} 15)$ y el testigo $\left(34,15^{\circ} \mathrm{C}\right.$ y 34,20 $\left.{ }^{\circ} \mathrm{C}\right)$. Lo cual obedeció a que la adición del residuo de ceniza mejoró las condiciones del suelo y así 
generó un medio óptimo para el desarrollo de la planta. Algunos investigadores señalan que existe una correlación entre el grado de apertura de los estomas con la luz, el contenido de agua en el suelo, la humedad relativa y la temperatura ambiental. Hartley 1988, Cayón 1998, Corley y Tinker 2009, Rivera 2009 y Peláez et al. 2010, Rivera et al. 2013 y López 2014.

\section{Conclusiones}

Al analizar el efecto de la incorporación de las diferentes dosis del residuo industrial de ceniza en el suelo para la corrección de la acidez intercambiable, no se logró una reacción estadísticamente significativa en el incremento del pH y la disminución de la acidez intercambiable en el suelo. Al incorporar $10000 \mathrm{~kg} \mathrm{ha}^{-1}$ de ceniza se obtuvo una respuesta positiva a los 90 dda en el crecimiento de las raíces terciarias y cuaternarias. A su vez, al adicionar $5000 \mathrm{~kg} \mathrm{ha}^{-1}$ y $15000 \mathrm{~kg} \mathrm{ha}^{-1}$ de este residuo se mostró una tendencia superior en el crecimiento y desarrollo de las raíces terciarias y cuaternarias a los 180 dda, causando un consecuente aumento en la densidad de raíz de las palmas.

Seencontró que la adición de ceniza en las plantas muestra una respuesta fisiológica en el aumento de la conductancia estomática.

\section{Referencias}

Albertazzi, H., Chinchilla, C., \& Ramírez, C. (2009). Dinámica del sistema radical de la palma aceitera (Elaeis guineensis Jacq.) en respuesta a la fertilización mineral y orgánica en suelos de áreas afectadas por pudriciones del cogollo. Oil Palm Papers. (33), 40-47.

Alvarado, A., Mata, R., \& Chinchilla, M. (2014). Arcillas identificadas en suelos de Costa Rica a nivel generalizado durante el período 1931-2014: Historia, metodología de análisis y mineralogía de arcillas en suelos derivados de cenizas volcánicas. Agronomía Costarricense. 38(1), 75106.

Arnone, J. (1997). Temporal responses of community fine roots populations to long term elevated atmospheric $\mathrm{CO} 2$ and soil nutrient patches in model tropical ecosystems. Acta Oecologica 18(3), 367-376. 
Bellote, A., Ferreira, C., Da Silva, H., \& Andrade, G. (1995). Efecto de la aplicación de ceniza de caldera y residuo de celulosa en el suelo y en el crecimiento de Eucalyptus grandis. Bosque. 16(1), 95-100.

Bidwell, R. (1993). Fisiología Vegetal. 1 ed. Ontario, Canadá. A.G.T Editor S.A.

Breure, J. (2003). The search for yield in oil palm: basic principles. In: fairhust, T. \& Hardter, R. (Eds.). Oil palm. Management for large and sustainable yields, (pp. 59-98). Oxford. Potash \& Phosphate Institute.

Burgos, C., Perdomo, R., Morales, C., \& Cayón, D. (1998). Efecto de los niveles de agua en el suelo sobre la palma de aceite (Elaeis guineensis Jacq.). II. Estado hídrico diario de palmas en etapa de vivero. Palmas. 19(2), 37-44.

Campbell, A. (1990). Reciclaje y eliminación de cenizas de madera. Tappi Journal. 73(9), 141-146.

Castro, H., \& Munevar, O. (2013). Mejoramiento químico de suelos ácidos mediante el uso combinado de materiales encalantes. Revista U.D.C.A Actualidad \& Divulgación Científica 16 (2), $409-416$.

Cayón, G. (1998). Ecofisiología de la palma de aceite (Elaeis guineensis Jacq.). Cenipalma, Primer Curso Internacional de Palma de Aceite (pp. 38-54). Bogotá, Colombia.

Chaimsohn, F., Montiel, M., Villalobos, E., \& Mora, J. (2008). Anatomía micrográfica del folíolo de la palma neotropical Bactris gasipaes (Arecaceae). Biol. Trop. 56(2), 952-959.

Charlton, W. (1997). Lateral root initiation. In: Plant roots the hidden half. Second Edition. Edited by Waisel, Y., Eshel, A. \& Kafkafi, U. Marcel Dekker.

Clapham, W., \& Zibilske, L. (1992). Wood ash as a liming amendment. Communications in Soil Science and Plant Analysis (23), 1227-1229.

Corley, R., \& Tinker, P. (2009). La palma de aceite. 4 ed. Bogotá, Colombia.

Corley, R., \& Tinker, P. (2003). The Oil Palm. 4 ed. Oxford, Reino Unido. Blackwell Science Ltd.

Corley, R. (1982). Fotosíntesis y Productividad. Amsterdam, Estados Unidos. Elsevier Scientific. 
Cristancho, J., Munévar, F., Acosta, A., Sancruz, L., \& Torres, M. (2007). Relación de las características edáficas y el desarrollo del sistema de raíces de la palma de aceite (Elaeis guineensis Jacq). Palmas. 28(1), 21-29.

Cruz-Ruiz, E., Cruz-Ruiz, A., \& Aguilera-Gómez, L. (2012). Efecto en las características edáficas de un bosque templado por el cambio de uso de suelo. Tierra Latinoamericana 30(2), 189-197.

Dell' Amico, J., \& Morales, D. (2017). Comportamiento de la conductancia estomática de dos variedades de tomate cubanas en condiciones de campo y riego limitado. Cultivos Tropicales. 38(n. ${ }^{\circ}$ esp.), 137-144.

Downie, A., Van, Z., Kimber, S., Morris, S., Chan, K., Rust, J., Joseph, S., \& Cowie, A. (2009). Effects of biochar from slow pyrolysis of papermill waste on agronomic performance and soil fertility. (Tesis doctoral). University of New South Wales. DOI 10.1007/s11104-009-0050-x

Escalante, A., Pérez, G., Hidalgo, C., Lopez, J., Campo, J., Valtierra, E., \& Etchers, J. (2016). Biocarbón (biochar) I: Naturaleza, historia, fabricación y uso en el suelo. Terra Latinoamericana 34(3), 367-382.

Escobar, R. (2012). ¿Por qué cultivar la palma aceitera?. Oil Palm Papers. (39), 13-26.

Etiégni, L., \& Campbell, A. (1991). Características físicas y químicas de ceniza de madera. Tecnología Bioambiental. 37(2), 173-178.

Galindo, T., \& Romero, H. (2012). Compostaje de subproductos de la agroindustria de palma de aceite en Colombia: estado del arte y perspectivas de investigación. Cenipalma. Bogotá, Colombia. Molher Impresores.

Garbanzo G., Molina E., Serrano E., \& Ramírez F. (2017). Efecto de mezclas de compost de fibra con suelo en el crecimiento de plántulas de palma aceitera en viveros y la incidencia de enfermedades foliares. Agronomía Costarricense. Costa Rica. 41(2), 47-66.

Guillén, C. (2013). Efectos de la aplicación de cenizas de caldera de biomasa en el modelo jerárquico de agregación de un suelo forestal bajo condiciones oceánicas. (Tesis licenciatura). Uiniversidad Autonoma de Barcelona. Derio, España. Recuperado de https://ddd.uab.cat/pub/trerecpro/2013/hdl_2072_210370/PFC_CarlaGuillenEscriba.pdf 
Gutierrez, M., Torres, J., \& Araya, J. (2014). Uso de winrhizo ${ }^{\circledR}$ en la cuantificación de las raíces y su aplicación en la palma aceitera (Elaeis guineensis jacq.)‥ Agronomía Mesoamericana. 25(1),181-188.

Hartley. C. (1988). The Oil Palm: Elaeis guineensis Jacq. 3 ed. Longman Group U.K. Limited. Londres, Inglaterra.

Hartley, C. (1983). La palma de aceite. Compañía Editorial Continental S.A. Mexico.

IICA (Instituto Interamericano de Cooperación para la Agricultura). (2016). Manejo de suelos ácidos de las zonas altas de honduras. Conceptos y métodos.Toledo, M. Tegucigalpa, Honduras. Editorial IICA.

IPNI (International Plant Nutrition Institute). (1999). Acidez y encalado de los suelos. La acidez del suelo.

Recuperado

de

http://www.cia.ucr.ac.cr/pdf/libros/Acidez\%20y\%20encalado\%20de\%20suelos,\%20libro\% 20por\%20\%20J\%20Espinosa\%20y\%20E\%20Molina.pdf

Jourdan C., \& Rey, H. (1997). Modelling and Simulation of the architecture and development of the oil palm Elaeis guineensis Jacq root system. 1. The model. PI. Soil. (190), 217-233.

Lallana, V. (2004). Fisologia Vegatal. Recuperado de www.fca.uner.edu.ar/files/academica/deptos/catedras/WEBFV_2010/mat_did/UT1_FV.p df

Lerner, B., \& Utzinger, J. (1986). Wood ash as soil liming material. Hortscience 21(1), 76-78.

López, J. (2014). Caracterización fisiológica y morfológica de palmas de aceite taisha (Elaeis oleífera HBK Cortés) y sus híbridos (Elaeis oleífera HBK Cortés x Elaeis guineensis Jacq.) en la región amazónica del ecuador. (Título de Maestría). Universidad Nacional de Colombia. Bogota D.C., Colombia. Recuperado de https://core.ac.uk/download/pdf/77273105.pdf

Madrigal, A., \& Garbanzo, G. (2018). Uso de residuos agroindustriales en previveros de palma aceitera (Elaeis guineensis, Arecaceae): crecimiento y absorción de nutrimentos. Cuadernos de Investigación, UNED. 10(2), 257-266. 
Merino, A., Otero, V., Omil, B., Lastra, B., Piñeiro, V., \& Gallego, P. (2006). Application of wood ash compared with fertigation for improving the nutritional status and fruit production of kiwi vines. J. Plant Nutr. Soil Sci. (169), 127-133.

Melado, A. (2008). Modelo de cultivo de palma aceitera (Elaeis guineensis Jacq.) en Honduras. (Tesis de grado). Universidad Politecnica de Madrid. Madrid, España. Recuperado de http://oa.upm.es/1671/1/PFC_ANGELA_MELADO_HERREROS.pdf

Odeoluwa, O., \& Adeoye, O. (2008). Potential of oil palm empty fruit bunch (EFB) as fertilizer in oil palm (Elaeis guineensis jacq) nurseries. Recuperado de http://orgprints.org/12808/

Ohno T., \& Erich, M. (1990). Efecto de la aplicación de cenizas de madera sobre el pH del suelo y los niveles de nutrientes de la prueba del suelo. Agricultura, Ecositemas y Medio ambiente. $32(3), 223-239$.

Ojeda, C. (2015). Efecto de un producto bioactivo compuesto por oligogalacturónidos como mitigador del estrés hidrico en varidedades de albahaca (Ocimun basilicum L). (Tesis Doctoral). Centro de Investigaciones Biologicas del Noroeste, S.C. Baja California Sur Granada, Mexico. Recuperado de https://cibnor.repositorioinstitucional.mx/ispui/bitstream/1001/170/1/ojeda c.pdf

Olsson, B., \& Kellner, O. (2002). Effects of soil acidification and liming on ground flora establishment after clear-felling of Norway spruce in Sweden. Forest Ecology and Management (158), 127139.

Omil, B. (2007). Gestion de cenizas como fertilizante y enmendante de plantaciones jovenes de Pinus radiata. (Tesis doctoral). Universidad de Santiago de Compostela. Lugo, España. Recuperado de https://minerva.usc.es/xmlui/bitstream/handle/10347/2374/9788497509640_content.pdf ;jsessionid=E5EE7C3B0FDEE69668674D30F9AE12C3? sequence $=1$

Othman, H., Tarmizi, A., \& Tayeb, M. (2005). Bunch ash: an efficient and cost-effective K fertilizer source for mature oil palm on peate under high rainfall environment. mpob Information.

Posso, M. (2010). Evaluación de diferentes dosis de compost y lombricompuesto aplicado al suelo de vivero de palma aceitera (Elaeis guineensis). (Tesis de grado). Universidad Nacional de Colombia. Valledupar, Colombia. 
Quirantes, M. (2015). Propuestas para la reutilización agrícola de cenizas generadas en la obtención de energias renovables a partir de biomasa residual. (Tesis Doctoral). Universidad de Granada. Granada, España. Recuperado de https://dialnet.unirioja.es/servlet/tesis?codigo $=57768$

Ramírez, N., Silva, A., Garzón, E., \& Yáñez, E. (2011). Boletín Técnico Nº 30. Caracterización y manejo de subproductos del beneficio del fruto de palma de aceite. Bogotá, Colombia, Cenipalma.

Raygada, R. (2005). Manual Técnico para el cultivo de la palma aceitera. Comisión Nacional para el Desarrollo y Vida sin Drogas. PRODATU.

Rees, A. (1961). Midday closure of stomata in the oil palm, Elaeis guineensis Jacq. Journal of Experimental Botany. (12), 129-146.

Reyes, R., Bastidas, S., \& Peña, E. (1997). Distribución del sistema radical de la palma de aceite (Elaeis guineensis Jacq.) en Tumaco, Colombia. Palmas. 18(3),49-57.

Rivera, Y., \& López, C. (2013). Physiological and morphological characterization of American oil palms (Elaeis oleifera HBK Cortes) and their hybrids (Elaeis oleifera $x$ Elaeis guineensis) on the indupalma. Agronomía Colombiana 31(3), 316-325.

Rivera, Y. (2009). Caracterización fisiológica y morfológica de palmas americanas de aceite (Elaeis oleífera H.B.K. Cortés) y sus híbridos (Elaeis oleífera x Elaeis guineensis) de la plantación Indupalma. (Tesis de Licenciatura). Universidad Nacional de Colombia. Bogotá, Colombia.

Rojas, O. (2015). Caracterización preliminar morfológica y fisiológica de 16 materiales de palma de aceite (Elaeis spp.) (Tesis de licenciatura). Universidad de los Llanos. Villavicencio, Colombia. Recuperado de https://repositorio.unillanos.edu.co/bitstream/001/366/3/Tesis\%20articulo\%20revista\%2 Oorinoquia.pdf

Romero, H., Ayala, I., \& Ruiz, R. (2007). Ecofisiologia de la palma de aceite. Palmas. 28(n. ${ }^{\circ}$ esp.), 176184.

Sanchez, V. (2003). La produccion de aceite de palma en méxico: caso estado de campeche. (Tesis de licenciatura). Universidad Autonama Agraria Antonio Narro. Coahuila, Mexico. Recuperado de http://usi.earth.ac.cr/glas/sp/Unarrow/0237.pdf 
Sánchez, M., \& Aguirreolea, J. (2000). Capítulo 3: Movimientos estomáticos y transpiración. Fisiología y bioquímica vegetal. 1 ed. McGraw-Hill Interamericana. Madrid, España.

Sierra, J., Sierra, L., \& Olivero, J. (2017). Potencial económico de la palma aceitera (Elaeis guineensis Jacq)1. Agronomía Mesoamericana. 28(2), 523-534.

Solla-Gullón, F., Taboada, M., Rodríguez-Soalleiro, R., \& Merino, A. (2004). Respuesta inicial del aporte de cenizas de biomasa arbórea en el estado nutricional de una plantación joven de Pinus radiata D. Don. Invest Agrar: Sist Recur For. 13 (2), 281-293.

Someshwar, A. (1996). Caracterización de la madera y la combinación de cenizas de calderas de leña. Revista de Calidad Ambiental. 25(5), 962-972.

Torres J. (2013). Caracterización morfo-fisiológica de palmas aceiteras (Elaeis guinensis Jacq.) afectadas por la pudrición del cogollo (PC) en Costa Rica. (Tesis de Licenciatura). Universidad de Costa Rica. San José, Costa Rica.

Torres, R., Acosta, A., \& Chinchilla, C. (2004). Proyecto Comercial de Compostaje de los desechos agroindustriales de la palma aceitera. Palmas. 25(n. ${ }^{\circ}$ esp.), 377-387.

Trucchi, G. (2015). La vertiginosa expansión de la palma africana. Recuperado de https://www.alainet.org/es/articulo/168452

Väätäinen, K., Sikanen, L., \& Asikainen, A. (2000). Rakeistetum puutunhkan metsäänpalautuksen logística. (Logistics of returning granulated wood ask bark to forest). University of Joensuu, Faculty of Forestry, Research Notes 116. Joensuu 2000.

Vance, E. (1996). Land Application of Wood-Fired and Combination Boiler Ashes: An Overview. J. Environ. Qual. (25), 937-944.

Zakaria, Z. (1998). Manejo de suelos y fertilizantes en plantaciones de palma de aceite en Malasia". Palmas. 25(n. ${ }^{\circ}$ esp.), 207-217. 